\title{
Immune thrombocytopenic purpura and intracranial haemorrhage: A formidable medical challenge
}

\author{
Chrystal Calderon* and A Perez \\ Department of Surgery (Neurosurgery), Eric Williams medical Sciences Complex, Champs Fleurs, Trinidad and Tobago
}

\begin{abstract}
Introduction: To discuss the thought-provoking and difficult clinical management of a case of spontaneous intracerebral haemorrhage and immune thrombocytopenic purpura (ITP).

Case presentation: A 6-year-old female, known ITP, presented with a one-day history of traumatic dislodged tooth, with associated neurological symptoms. However, her neurological status was alert and oriented, with absence of focal deficits. The complete blood count showed a platelet count of $26 \mathrm{x} 10^{9} / \mathrm{L}$ and was in keeping with her known baseline platelet level. Computed tomography of the brain demonstrated three sites of cerebral contusions with no associated midline shift.

Hours after admission, there was progressive decline in the patient's clinical status. Additionally, repeat blood investigations showed a downward trend in platelet counts. Aggressive medical management was implemented with the aid of neurosurgery, haematology, paediatrics, and the intensive care unit. Despite various therapeutic modalities, the patient succumbed to her underlying disorder.

Conclusion: On retrospective review, this patient had a severe phenotype of ITP. This was demonstrated by repeated oral mucosa bleeds, gastrointestinal bleeds and episodes of haematuria preceding this last admission. Despite multimodal therapies and the combined efforts of a multi-disciplinary team, the clinical management remained arduous.
\end{abstract}

Abbreviations: ITP: Immune thrombocytopenic purpura; GCS: Glasgow coma scale

\section{Introduction}

Thrombocytopenia may result from an impairment in platelet production, an increase in platelet consumption and splenic sequestration [1]. ITP is thought to occur following a viral illness and may be self- limiting in children [2]. Traditional terminology categorised the pathological course into acute and chronic phases. It may also be classified as newly diagnosed, persistent or chronic $[3,4]$. This case patient fell into the group of persistent- which is within 312 months following diagnosis. Her initial diagnosis was established 6 months prior to this ultimate presentation, with prior recurrent visits to the emergency department with bruising over the body, conjunctival haemorrhages, and a lower gastro-intestinal bleed.

We believe there were combined underlying aetiologies- of trauma and spontaneous elements. There was a known history of minor blunt trauma however, the pattern of haemorrhages on imaging is not consistent with an isolated traumatic injury. Spontaneous nontraumatic intracerebral haemorrhage in the paediatric population is far less common relative to the adult population. ITP will result in cerebral haemorrhage in $0.1 \%-1 \%$ of cases, and the majority of published cases demonstrate that this will occur within the first 6 months of diagnosis [5].

\section{Case history}

A 6-year-old Afro-Caribbean female, known ITP, presented with a one-day history of a traumatic dislodged tooth with persistent bleed. Approximately 24 hours following the injury, the patient started complaining of headaches. The headaches were described as mild to moderate and was associated with nausea and 2 episodes of vomiting.

On initial examination, her Glasgow coma scale (GCS) was 15, with pupils equal and reactive bilaterally. A complete blood count revealed a platelet count of $26 \times 10^{9} / \mathrm{L}$ and a computed tomography of the brain without contrast was performed. It showed the presence of the three distinct foci of hyper-densities in keeping with, cerebral contusions. A left frontal lobe contusion of $2.6 \mathrm{~cm} \times 2.3 \mathrm{~cm}$, a right frontal lobe contusion of $1.8 \mathrm{~cm} \mathrm{x} 1.5 \mathrm{~cm}$ and left parietal lobe contusion $1.8 \mathrm{~cm} \mathrm{x}$ $2.3 \mathrm{~cm}$. There was no evidence of midline shift and the dimensions of the ventricular system remained within normal limits (Figures 1 and 2).

Supportive medical management and neuro- observation was commenced with the support of haematology and paediatric medicine. An altered conscious level was noted 12 hours following admission, with a drop in GCS to 9. An urgent blood panel was requested showing a platelet count of $18 \times 10^{9} / \mathrm{L}$. Computed tomography of the brain was repeated. It showed a moderate increase in size of the cerebral contusions. The left frontal lobe contusion measured $3.3 \mathrm{~cm} \times 2.7 \mathrm{~cm}$, right frontal lobe contusion $2.2 \mathrm{~cm} \times 1.9 \mathrm{~cm}$ and left parietal lobe contusion $2.3 \mathrm{~cm} \times 2.7 \mathrm{~cm}$. There was no associated midline shift or ventricle effacement once more (Figure 3 ).

${ }^{*}$ Correspondence to: Chrystal Calderon, Department of Surgery, EWMSC, Champs Fleurs, Trinidad and Tobago, Tel: 1(868)460-0339; E-mail: ccalderon88@gmail.com

Key words: immune thrombocytopenic purpura (ITP), intracerebral haemorrhage, medical management, neurosurgical intervention

Received: July 01, 2020; Accepted: July 07, 2020; Published: July 10, 2020 


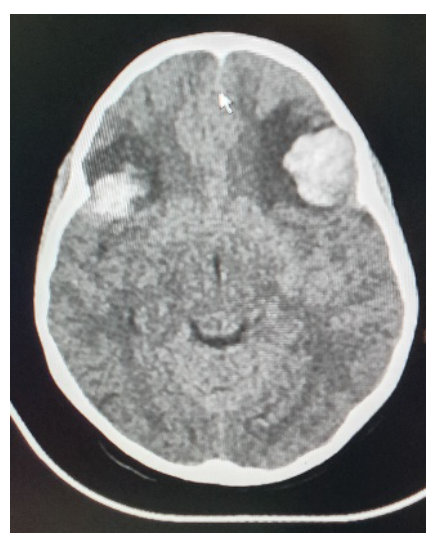

A

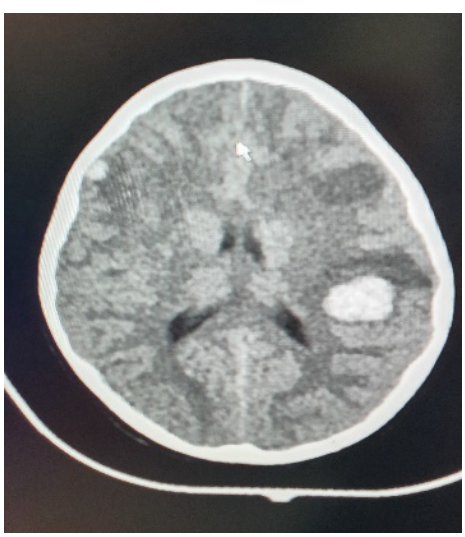

B
Figure 1. Computed tomography of the brain (axial view) showing A. cerebral haemorrhage in bifrontal regions and B. cerebral haemorrhage in left parietal region

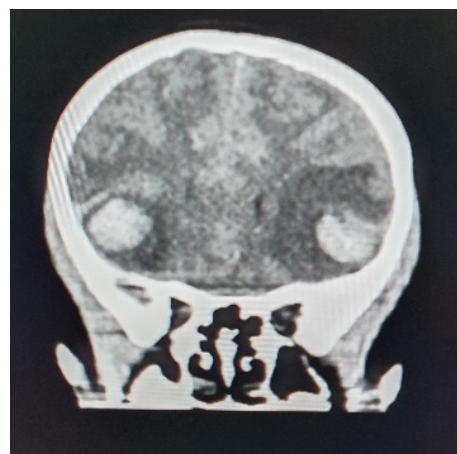

A

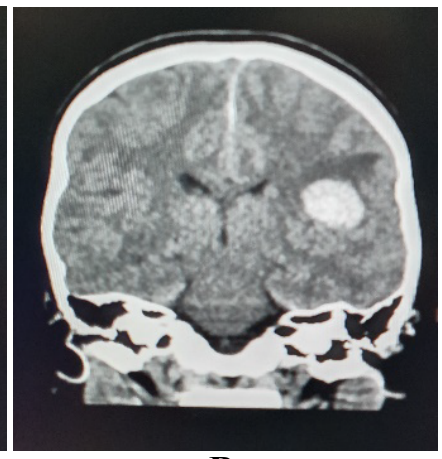

B
Figure 2. Computed tomography of the brain (coronal view) showing A. cerebral haemorrhage in bifrontal regions and B. cerebral haemorrhage in left parietal region
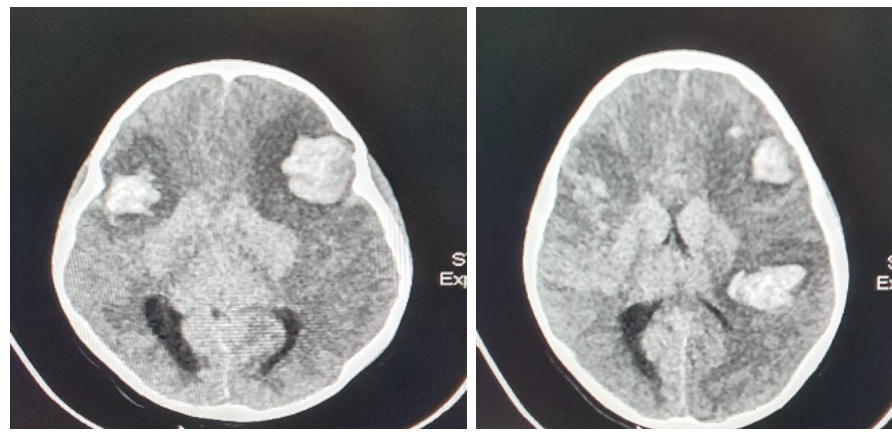

Figure 3. Repeated computed tomography of the brain (axial view) showing increased size of cerebral haemorrhages in bilateral frontal regions and left parietal region, with perilesional edema

New clinical manifestation of seizures presented within the next couple of hours, and the patient remained in status epilepticus for approximately 45 minutes. Examination findings showed a dilated $(5 \mathrm{~mm})$, unreactive left pupil and a reactive, $3 \mathrm{~mm}$ right pupil. Her vital signs were deranged with elevated systolic blood pressures (123-132 mmHg), tachypnoea and bradycardia (56 - 60 beats per minute). Neurosurgical intervention was planned with a bi-frontal decompressive craniectomy to aid with the increasing cerebral edema. Paediatric intensive care physicians and anaesthesiologists were asked to consult on the case. The ultimate decision was for resuscitative methods - including endotracheal intubation, additional sedative and anticonvulsant medications and achieving a suitable platelet count of 50- $10010^{9} / \mathrm{L}$ for safe surgical intervention.
Attempts at improving the platelet count failed over the upcoming days. The patient was transfused with a total of 18 units of platelets. She was also administered intravenous immune globulins, corticosteroid medication and tranexamic acid. The platelet count remained low, fluctuating between $11-14 \times 10^{9} / \mathrm{L}$. The course in paediatric intensive care unit was complicated with the development of central diabetes insipidus and radiological evidence of a right pulmonary haemorrhage. Her GCS was assessed as 3 and pupils were dilated and unreactive bilaterally on day 3 following admission. She underwent initial brainstem death testing and unfortunately succumbed to her condition on day 8 .

\section{Discussion}

ITP is an uncommon haematological condition occurring in both the adult and paediatric age groups. The incidence in adults and children are estimated as 0.2- 0.4 new cases per 10,000/annum and $0.2-0.7$ new cases per 10,000/ annum, respectively [4]. The pathology of the disease stems from the imbalance of platelet consumption and production. An autoimmune condition develops where autoantibodies are formed against platelets and their precursor, megakaryocytes. Autoantibodies binding to the platelet membrane proteins leads to direct damage and promotes the removal of these platelets from circulation by phagocytosis in the liver and spleen [6]. Megakaryocyte depletion impairs the production of new platelets in the bone marrow and hence, cannot compensate for the depletion taking place in the circulatory system [6,7].

Based on this background, it is clear that these patients will tend to bleed even from minor injury. These include the presence of easy bruising over the limbs and trunk, bleeding from mucus membranes, and difficult to control bleeds from traumatic injury. ITP is diagnosed based on laboratory blood investigation demonstrating an isolated thrombocytopenia of less than $100 \times 10^{9} / \mathrm{L}$ and an absence of any underlying predisposing conditions [8]. The normal platelet count usually lies within the range of $150 \times 10^{9} / \mathrm{L}$ and $450 \times 10^{9} / \mathrm{L}$; a count between $10-20 \times 10^{9} / \mathrm{L}$ places a patient at risk for spontaneous haemorrhage [1,3]. Based on World Health Organisation (WHO) bleeding scale this case patient falls into the highest category, Grade 4 - with central nervous system bleeding on imaging study. This is denoted as life-threatening, with a likelihood of permanent functional impairment [9-11]. Fortunately, the probability of a fatal bleed or a severe bleed in a child is low - with an evaluation of $0 \%-3 \%[4,8]$.

In children, some of the common associations of spontaneous intracerebral haemorrhage include arteriovenous malformations, cavernous angiomas, cerebral aneurysms, brain tumours and haematological conditions [12,13]. The latter, which includes coagulopathies, sickle cell disease and hemophilias, represent a substantial risk factor in approximately $10-30 \%$ of cases $[5,14]$. The most common cerebral site affected is the supra-tentorial region, with lobar haemorrhages. A poor prognostic outcome in these cases are directly correlated to the site of haemorrhage as with infratentorial bleeds, a low GCS on presentation, and younger children ( $<3$ years) $[12,15,16]$.

Surgical intervention was considered in this case patient, with a possible bifrontal craniotomy to attenuate the effects of an elevated intracranial pressure. However, attempts at optimization for safe surgical intervention faired unfavourable with falling platelet counts. This is despite the use of several units of platelets, and a combination of drugs- intravenous immunoglobulins, high dose corticosteroid therapy and tranexamic acid - as recommended $[17,18]$. Further steps 
to be considered was an emergency splenectomy, which has shown to lead to a sustained rise in platelet count post operation [19]. We believe following through with a craniectomy or splenectomy in this thrombocytopenic state would have led to devastating intra-operative complications.

The presence of an intracranial haemorrhage in ITP has devastating mortality rates, with estimations of $25 \%-55 \%$ [5,20]. Additionally, there is noteworthy neurological morbidity that may persist in non-fatal cases. This was a challenging neurosurgical and haematological case to manage and it could not be done in isolation. It required the combined efforts and decision-making of neurosurgeons, haematologists, physicians, and anaesthetists. Unfortunately, the patient succumbed to the sequelae of events despite these efforts.

\section{Conclusion}

Intracranial haemorrhage resulting from ITP in the paediatric population is not frequently seen in clinical practice. It carries significant morbidity and mortality even with urgent aggressive management- usually requiring a multi-disciplinary team. Our case patient proved to be refractory to combination drug therapy and platelet transfusions with no notable rise in platelet levels during her course in-hospital. This case was a medical challenge in optimisation for safe surgical intervention.

\section{Conflict of interest}

There are no conflicts of interest to be stated.

\section{References}

1. Izak M, Bussel J (2018) Management of thrombocytopenia. Rev Med Brux.

2. Lambert MP, Gernsheimer TB (2017) Clinical updates in adult immune thrombocytopenia. Blood 129: 2829-2835.

3. Kayal L, Jayachandran S, Singh K (2014) Idiopathic thrombocytopenic purpura. Contemp Clin Dent 5: 410-414.

4. Matzdorff A, Meyer O, Ostermann H, Kiefel V, Eberl W, et al. (2018) Immune Thrombocytopenia - Current Diagnostics and Therapy: Recommendations of a Join Working Group of DGHO, ÖGHO, SGH, GPOH, and DGTI. Oncol Res Treat 41: $1-30$.
5. Jordan LC, Hillis AE (2007) Hemorrhagic Stroke in Children. Pediatr Neurol 36: 73-80

6. Kuwana M, Okazaki Y, Kaburaki J, Kawakami Y, Ikeda Y (2002) Spleen Is a Primary Site for Activation of Platelet-Reactive $\mathrm{T}$ and $\mathrm{B}$ Cells in Patients with Immune Thrombocytopenic Purpura. J Immunol 168: 3675-3682.

7. Khan M, Mikhael J (2010) A review of immune thrombocytopenic purpura: focus on the novel thrombopoietin agonists. J Blood Med 1: 21-31.

8. Farhangi H, Ghasemi A, Banihashem A, Badiei Z, Jarahi L, et al. (2016) Clinical Features and Treatment Outcomes of Primary Immune Thrombocytopenic Purpura in Hospitalized Children Under 2-Years Old. Iran J Pediatr Hematol Oncol 6: 24.

9. Miller AB, Hoogstraten B, Staquet M, Winkler A (1981) Reporting results of cancer treatment. Cancer 47: 207-214.

10. Estcourt LJ, Heddle N, Kaufman R, McCullough J, Murphy MF, et al. (2013) The challenges of measuring bleeding outcomes in clinical trials of platelet transfusions. Transfusion 53: 1531-1543.

11. US Department of Health and Human Services, National Institute of Health, National Cancer Institute. CTCAE (Common Terminology Criteria for Adverse Event) v5.0. National Cancer Institute Division of Cancer Treatment and Diagnosis. 2018.

12. Lin CL, Loh JK, Kwan AL, Howng SL (1999) Spontaneous intracerebral hemorrhage in children. Kaohsiung J Med Sci 15: 146-151.

13. Kumar R, Shukla D, Mahapatra AK (2009) Spontaneous intracranial hemorrhage in children. Pediatr Neurosurg 45: 37-45.

14. Blom I, De Schryver ELLM, Kappelle LJ, Rinkel GJE, Jennekens-Schinkel A, et al (2003) Prognosis of haemorrhagic stroke in childhood: A long-term follow-up study Dev Med Child Neurol 45: 233-239.

15. Abbas Q, Merchant QUA, Nasir B, Haque AU, Salam B, et al. (2016) Spectrum of Intracerebral Hemorrhage in Children: A Report from PICU of a Resource Limited Country. Crit Care Res Pract.

16. Meyer-Heim AD, Boltshauser E (2003) Spontaneous intracranial haemorrhage in children: Aetiology, presentation and outcome. Brain Dev 25: 416-421.

17. Arnold DM (2015) Bleeding complications in immune thrombocytopenia. Hematology 2015: 237-242.

18. Khan AM, Mydra H, Nevarez A (2017) Clinical Practice Updates in the Management of Immune Thrombocytopenia. P T.

19. Iyori H, Bessho F, Ookawa H, Konishi S, Shirahata A, et al. (2000) Intracranial hemorrhage in children with immune thrombocytopenic purpura. Ann Hematol 79: 691-695.

20. Psaila B, Petrovic A, Page LK, Menell J, Schonholz M, et al. (2009) Intracranial hemorrhage (ICH) in children with immune thrombocytopenia (ITP): Study of 40 cases. Blood 114: 4777-4783.

Copyright: (C2020 Calderon C. This is an open-access article distributed under the terms of the Creative Commons Attribution License, which permits unrestricted use, distribution, and reproduction in any medium, provided the original author and source are credited. 